\title{
Estudo do nível de desenvolvimento da coordenação motora da população escolar (6 a 10 anos de idade) da Região Autónoma dos Açores*
}

V.P. Lopes ${ }^{1}$

J.A.R. Maia ${ }^{2}$

R.G. Silva ${ }^{2}$

A.Seabra ${ }^{2}$

F.P. Morais ${ }^{2}$

https://doi.org/10.5628/rpcd.03.01.47

\section{RESUMO}

Os propósitos da presente investigação foram: (1) caracterizar o estado de desenvolvimento da coordenação motora ao longo dos quatro anos do $1^{\circ}$ ciclo do ensino básico (1CEB); (2)

mapear as diferenças entre as crianças dos dois sexos; e (3) identificar a presença de insuficiência de desenvolvimento coordenativo. A amostra foi constituída por 3742 crianças de ambos os sexos dos 6 aos 10 anos de idade a frequentar o 1CEB na Região Autónoma dos Açores. A coordenação foi avaliada através da bateria de testes de coordenação corporal

(Körperkoordinationstest für Kinder - KTK) que é composta por quatro testes: (1) equilíbrio em marcha à retaguarda (ER); (2) saltos laterais (SL); (3) saltos monopedais (SM); e (4) transposição lateral (TL). A partir do resultado de cada teste, e através da utilização das tabelas de valores normativos fornecidas pelo manual, obtém-se um quociente motor (QM). Fez-se a análise teste a teste e ao QM. Para além das medidas descritivas (média e desvio-padrão), recorreu-se à ANOVA factorial para identificar diferenças entre os sexos ao longo da idade, e utilizou-se a função discriminante para testar a presença de perfis multivariados de coordenação motora. Verificou-se que, em ambos os sexos e em todas as provas da bateria, ocorre um incremento significativo dos valores médios de cada teste ao longo da idade, tendo os meninos valores médios superiores aos das meninas em todos os intervalos etários em todos os itens da bateria, com a excepção dos SL. Os valores médios do desempenho nas quatro provas são inferiores aos valores médios obtidos noutros estudos realizados, quer em Portugal, quer no estrangeiro. Contrariamente ao esperado, os valores médios do QM decrescem com a idade. A generalidade das crianças, em ambos os sexos e nos diferentes intervalos etários, situa-se no intervalo de insuficiência coordenativa e de perturbações de coordenação. Constata-se, em ambos os sexos, a tendência generalizada para as crianças de uma idade mostrarem perfis de coordenação motora inferiores àqueles que são esperados para a sua idade. Tal circunstância revela uma forte insuficiência em aspectos do desenvolvimento coordenativo nas diferentes idades.

Palavras-chave: coordenação motora, diferenças entre sexos, perfis multivariados.

\author{
${ }^{1}$ Instituto Politécnico de Bragança, Portugal \\ ${ }^{2}$ Faculdade de Ciências do Desporto e de Educação Física \\ Universidade do Porto, Portugal.
}

\begin{abstract}
Motor coordination level of school aged children (6-10 years) of the Azores Islands

The purposes of this investigation were: (1) to characterize the developmental state of motor coordination in primary school children; (2) to identify differences between children of both genders; and to (3) identify the presence of insufficient motor coordination. Sample comprises 3742 children of both genders with 6 to 10 years of age. Motor coordination was evaluated with the test battery "Körperkoordinationstest für Kinder" (KTK). The battery comprises four tests: backward balance (ER), jumping sideways (SL), hopping on one leg (SM), and shifting platforms (TL). Factorial ANOVA (gender*age) was used to identify differences between boys and girls in each age. Discriminant function was used to test the presence of multivariate profiles of motor coordination. It was found a performance increment with age in both genders in all items of test battery, and that boys had higher mean values than girls in all ages and in all items of the test battery, but in SL. Mean values of performance in all test items were lower than in other Portuguese and foreigner samples. Most of the children of both genders in all ages had a motor quotient in the interval of low coordination and coordination disorder. In both genders was noted a tendency for children of one age to have a lower profile of motor coordination than the one expected for their own age. These results showed that children of all ages had a low development of motor coordination.
\end{abstract}

Key Words: motor coordination, gender differences, multivariate profiles. 


\section{INTRODUÇÃO}

Segundo Kiphard e Schilling (4) coordenação é a interacção harmoniosa e económica do sistema músculo-esquelético, do sistema nervoso e do sistema sensorial com o fim de produzir acções motoras precisas e equilibradas, e reacções rápidas adaptadas a situações que exigem:

1) uma adequada medida de força que determina a amplitude e velocidade do movimento;

2) uma adequada selecção dos músculos que influenciam a condução e orientação do movimento;

3) a capacidade de alternar rapidamente entre tensão e relaxação musculares.

O conceito de coordenação motora é abordado em diferentes âmbitos, contextos e áreas científicas (controlo motor, aprendizagem motora, desenvolvimento motor, biomecânica, fisiologia, etc.). De facto, a coordenação motora pode ser analisada segundo três pontos de vista: (1) biomecânico, dizendo respeito à ordenação dos impulsos de força numa acção motora e a ordenação de acontecimentos em relação a dois ou mais eixos perpendiculares; (2) fisiológico, relacionando as leis que regulam os processos de contracção muscular; (3) pedagógico, relativo à ligação ordenada das fases de um movimento ou acções parciais e a aprendizagem de novas habilidades (11). No contexto da presente investigação é neste último ponto de vista que nos situamos, tal como sugerido por Kiphard e Schilling (4).

A abordagem de Schilling e Kiphard adequa-se bem ao contexto da presente investigação, apesar das suas pesquisas terem já mais de trinta anos. De facto, os autores que mais avançaram na operacionalização da coordenação motora foram Kiphard e Schilling $(3,4,5)$. Dos seus estudos, que tiveram preocupações sobretudo pedagógicas e clínicas, resultou uma bateria de testes para avaliar a coordenação motora de crianças dos 5 aos 14 anos de idade, designada por bateria de testes de coordenação corporal para crianças (Körperkoordinationtest für Kinder - KTK) (5). Após vários estudos empíricos, usando a análise factorial exploratória, foi identificado um factor designado por coordenação corporal que continha os quatro testes actuais da bateria KTK. Esta bateria continua sendo aquela a que se recorre para avaliar a coordenação motora grosseira e identificar crianças com insuficiência coordenativa.
O estudo da coordenação motora reveste-se de grande importância em várias disciplinas científicas como a aprendizagem motora, o controlo motor e o desenvolvimento motor. Estas disciplinas focam os seus esforços no sentido de entender como as acções motoras se processam a diferentes níveis, desde a forma como são reguladas até ao seu resultado. No contexto da presente investigação interessa-nos sobretudo identificar as crianças com problemas a este nível, as crianças que se designam por descoordenadas ou desajeitadas (awkward ou clumsy em língua inglesa). De facto, o que nos motiva é a necessidade de identificar, com alguma precisão, as crianças com debilidade motora ou insuficiência de coordenação.

A insuficiência de coordenação refere-se à instabilidade motora geral, que engloba os defeitos qualitativos da condução do movimento atribuído a uma interacção imperfeita das estruturas funcionais subjacentes, i.e., sensoriais, nervosas e musculares (3), a qual provoca uma moderada alteração qualitativa dos movimentos e produz uma diminuição leve a mediana do rendimento motor. Esta insuficiência de coordenação pode e deve ser corrigida por medidas adequadas no contexto da Educação Física escolar. A medida da insuficiência de coordenação é geralmente dependente da qualidade e quantidade de experiências motoras vivenciadas pelas crianças. Uma melhoria da capacidade de trabalho das crianças, com menor disponibilidade, parece ser possível desde que a causa seja a fraca experiência motora (sobre esta matéria ver 12).

Os estudos sobre esta matéria, tanto em Portugal como no estrangeiro, não são vastos. A generalidade das investigações dividem-se em dois tipos: (1) os estudos que procuram caracterizar uma dada população ou amostra quanto ao nível de desenvolvimento coordenativo, procurando determinar os seus preditores e (2) as pesquisas que analisam os efeitos do ensino no desenvolvimento da coordenação.

Apresentamos de seguida um apanhado geral destas investigações que têm em comum o facto de terem utilizado a bateria KTK para avaliar a coordenação motora grosseira.

\section{Resultados de estudos no estrangeiro}

O padrão de desenvolvimento da capacidade de coordenação corporal foi assumido por Kiphard e 
Schilling (5) como aumentando linearmente com a idade e de uma forma paralela em ambos os sexos, tendo o sexo feminino, relativamente ao sexo masculino, um resultado superior em 16 pontos aos 6,6 anos e em 10 pontos aos 10,6 anos. Estes resultados devem, no entanto, ser considerados com reservas, já que o método de estudo transversal usado pelos autores não é o mais adequado para este tipo de análise. Willimczik (14), num estudo longitudinal $(6,7$ aos 10,7 anos), verificou que os resultados contradiziam o padrão de desenvolvimento assumido por Kiphard e Schilling (5) em dois aspectos. Primeiro, não foram encontradas diferenças significativas entre os sexos nos cinco momentos de avaliação e, segundo, foi encontrada uma interacção significativa entre o factor tempo e o género sexual. Assim, tem que ser assumido um padrão de desenvolvimento específico de cada sexo e não um padrão de desenvolvimento invariante. Esta especificidade foi demonstrada pelo facto de os rapazes que tinham resultados inferiores às meninas aos 6,6 anos, obterem resultados melhores do que estas aos 8,6 anos.

Matinek, Zaichkowsky e Cheffers (11) verificaram os efeitos de modelos de ensino vertical e horizontal (de acordo com as decisões partilhadas pelo professor ou alunos), na coordenação avaliada através do teste KTK, em 600 crianças da escola elementar (do $1^{\circ}$ ao $5^{\circ}$ grau). Verificaram que os grupos com modelo de ensino vertical obtiveram resultados significativamente superiores aos grupos com modelo de ensino horizontal e aos grupos de controlo, e que as crianças mais velhas obtiveram resultados significativamente superiores às mais novas. Constataram, portanto, que o ensino tem efeitos benéficos e significativos sobre a coordenação motora.

Zaichkowsky, Zaichkowsky e Martinek (15) analisaram os efeitos de um programa de actividades físicas na coordenação em 299 crianças de 7 a 12 anos de idade. A amostra foi dividida em grupo experimental e de controlo. Ao grupo experimental foram leccionadas aulas de EF de 50 minutos, uma vez por semana, ao longo de 24 semanas. O grupo experimental obteve melhores resultados do que o grupo de controlo. Verificaram que os resultados vão melhorando com o aumento da idade. Os resultados indicam que a participação em actividades físicas organizadas tem efeitos positivos no desenvolvimen- to da coordenação em crianças de 7 a 11/12 anos, mesmo com apenas uma sessão semanal.

Leurs et al. (6) analisaram os efeitos de um programa de treino psicomotor em 38 crianças com doença cardíaca congénita, ao longo de oito meses, com uma sessão semanal de 1,5 horas. Usaram a bateria KTK para avaliar a capacidade de coordenação corporal antes e após a aplicação do programa. No préteste encontraram défices de coordenação em $63 \%$ das crianças. Após a aplicação do programa o quociente motor (resultado global da bateria KTK) aumentou significativamente. No pós-teste, $71 \%$ das crianças puderam ser classificadas como tendo um desenvolvimento da coordenação normal. Os resultados deste estudo dão ênfase à importância de programas especiais de treino/instrução em crianças com necessidades específicas.

\section{Estudos realizados em Portugal}

Tendo como objectivo analisar o efeito de aulas suplementares de Educação Física ao longo de um ano lectivo no desenvolvimento da coordenação em crianças de 10 e 11 anos de idade, Mota (13) realizou um estudo onde sujeitou um grupo experimental a um programa de aulas suplementares durante um ano lectivo, num total de 56 sessões de 50 minutos. O programa de aulas suplementares tinha como objectivo a compensação das insuficiências de natureza postural, coordenativa e orgânica. A avaliação da capacidade de coordenação corporal foi realizada através da bateria KTK. No final do ano lectivo registou uma melhoria generalizada do grupo experimental, especialmente na tarefa de equilíbrio à retaguarda. Andrade (1) realizou um levantamento dos níveis de coordenação motora de crianças $(n=315)$ de ambos os sexos na região autónoma da Madeira, tendo comparado os diferentes grupos etários em cada género sexual. Verificou que apenas aos 9 anos de idade existem diferenças significativas entre rapazes e raparigas nos níveis de desempenho em apenas dois testes (equilíbrio à retaguarda e saltos laterais). Constatou que o desempenho era sempre superior nos grupos etários de idade mais avançada relativamente aos de idade mais baixa, tal como já tinham verificado Kiphard e Schilling (5) e Willimczik (14). Com o intuito de caracterizar os níveis de coordenação motora das crianças de duas freguesias de 
Matosinhos (Matosinhos e Lavra), Gomes (2) avaliou 214 crianças de ambos os sexos nos intervalos etários de 8, 9 e 10 anos. Apurou que o desempenho, na generalidade, melhora com a idade em ambos os sexos. No entanto, aos 9 anos de idade verificou, através da análise da função discriminante, que uma grande percentagem era reclassificada no grupo etário de 8 anos. Quando comparou os resultados da amostra com os resultados de outros estudos, por ex. Kiphard e Schilling (5), constatou que as crianças de Matosinhos apresentavam desempenhos inferiores.

Lopes e Maia (9) analisaram a magnitude da mudança na expressão da capacidade de coordenação corporal em crianças de 8 anos de idade sujeitas a dois programas de ensino e a duas frequências lectivas semanais (2/semana e 3/semana) ao longo de um trimestre lectivo. Um dos programas de ensino consistiu no bloco jogos do programa oficial do 1CEB; o segundo programa consistiu numa unidade didáctica que tinha por base o basquetebol. A capacidade de coordenação corporal foi avaliada através da bateria de testes KTK. O estudo teve um delineamento factorial (programa $\mathrm{x}$ frequência $\mathrm{x}$ tempo). Verificaram que houve melhorias em todos os grupos. O programa oficial teve um maior efeito sobre a melhoria do item saltos laterais. Relativamente à frequência semanal não se podem tirar quaisquer conclusões, uma vez que os efeitos dos dois níveis de frequência são distintos nos dois itens onde este factor teve influência significativa (saltos laterais e transposição lateral).

Lopes (7) alargou o âmbito e os objectivos do estudo anterior no sentido de analisar a mudança ocorrida ao longo do ano lectivo na coordenação de crianças em idade escolar, quando sujeitas a diferentes programas e a diferentes frequências semanais de aulas de Educação Física. O estudo consistiu na aplicação de dois programas de Educação Física ao longo de um ano escolar: um elaborado a partir do programa oficial de Educação Física do 1CEB, e o outro, um programa alternativo, orientado pelo princípio de que a Educação Física das crianças é educação desportiva, baseado nas habilidades das seguintes modalidades desportivas: futebol, basquetebol, ginástica, atletismo e andebol. Cada programa foi aplicado com duas frequências semanais (2 e 3 aulas). No estudo participaram 5 turmas do 1CEB, num total 100 crianças de 9 anos de idade, reparti- das em 4 grupos experimentais e 1 grupo de controlo. A capacidade de coordenação corporal foi avaliada em três momentos, de acordo com a bateria KTK. Os resultados do estudo indicam que o desenvolvimento dos níveis de expressão da capacidade de coordenação corporal foi mais elevado nos grupos de crianças sujeitas a aulas de Educação Física (grupos experimentais) do que no grupo de controlo. Foram encontradas diferenças significativas entre os efeitos dos dois programas, tendo as crianças sujeitas ao programa alternativo obtido ganhos superiores às crianças sujeitas ao programa oficial. Verificaram diferenças significativas entre os efeitos das duas frequências semanais de aulas, tendo as crianças com 3 aulas semanais melhorado mais a sua prestação do que as crianças com 2 aulas semanais.

O estudo pedagógico e epidemiológico da capacidade de coordenação corporal permite, de certa forma, aferir do grau de desenvolvimento coordenativo das crianças e jovens e, numa segunda fase, sugerir medidas de intervenção para corrigir as insuficiências detectadas.

Os propósitos da presente investigação são pois: (1) caracterizar o estado de desenvolvimento da coordenação motora grosseira ao longo dos quatro anos do $1 \mathrm{CEB}$ das crianças da Região Autónoma dos Açores; (2) mapear as diferenças entre as crianças dos dois sexos; e (3) identificar a presença de insuficiência de desenvolvimento coordenativo.

\section{MATERIAL E MÉTODOS \\ Amostra}

A amostra foi constituída por 3742 crianças de ambos os sexos dos 6 aos 10 anos de idade a frequentar o 1CEB na Região Autónoma dos Açores. O Quadro 1 apresenta o número de sujeitos por faixa etária e por sexo.

\section{Quadro 1 - Estratificação da amostra (frequência absoluta em função da idade e género sexual]).}

\begin{tabular}{lccc} 
Idade & Masculino & Feminino & Total \\
\hline 6 & 269 & 286 & 555 \\
7 & 431 & 453 & 884 \\
8 & 428 & 464 & 892 \\
9 & 460 & 429 & 889 \\
10 & 241 & 281 & 522 \\
Total & 1829 & 1913 & 3742
\end{tabular}




\section{Avaliação da coordenação motora}

Para a avaliação da coordenação motora foi utilizada a bateria KTK (Körperkoordinationstest für Kinder KTK) (5), constituída por quatro itens: (1) equilíbrio em marcha à retaguarda (ER); (2) saltos laterais (SL); (3) saltos monopedais (SM); e (4) transposição lateral (SL).

O resultado de cada item é comparado com os valores normativos fornecidos pelo manual, sendo atribuído a cada item um quociente. O somatório dos quatro quocientes representa o quociente motor (QM) que pode ser apresentado em valores percentuais ou absolutos, permitindo classificar as crianças segundo o seu nível de desenvolvimento coordenativo: (1) perturbações da coordenação (QM < 70); (2) insuficiência coordenativa (71 $\leq$ QM £85); (3) coordenação normal (86 $\mathrm{QM} \leq 115)$; (4) coordenação boa (116 $\mathrm{QM} \leq 130)$; (5) coordenação muito boa $(131 \leq \mathrm{QM} \leq 145)$. A bateria KTK permite, portanto, dois tipos de análise dos resultados: (1) por prova ou (2) pelo valor global do QM.

\section{Procedimentos estatísticos}

Para além das medidas descritivas (média e desvio-padrão), recorreu-se à ANOVA factorial (sexo * idade) para identificar diferenças entre os sexos ao longo da idade.

Utilizou-se a análise da função discriminante para testar a presença de perfis multivariados de coordenação motora. Dos resultados obtidos com este procedimento multivariado, só consideraremos a parte que trata da reclassificação dos sujeitos com base nos valores esperados de coordenação em cada idade, e que se designa por matriz de reclassificação, ou de confusão.

\section{Controlo da qualidade dos dados}

O controlo dos dados passou por um conjunto variado de crivos por forma a garantir a maior qualidade possível da informação obtida:

- Em primeiro lugar, foram apresentados, da forma mais detalhada possível, o conteúdo e alcance do projecto, bem como os detalhes da estrutura da avaliação da coordenação motora. Os membros das equipas testaram, neles próprios, toda a estrutura da avaliação. Os problemas e questões inventariados nesta etapa foram solucionados pelos responsáveis da investigação.

- Em segundo lugar, foi realizado um estudo piloto pelas quatro equipas de avaliação em quatro ilhas, garantindo que a amostra a ser testada (aproximadamente 40 sujeitos em cada ilha) fosse dos dois sexos e tivesse alunos dos 4 anos de escolaridade. O Quadro 2 refere-se aos resultados deste estudo piloto e tem, no seu corpo, as estimativas de fiabilidade dos resultados da avaliação das crianças.

Quadro 2 - Estimativas de fiabilidade dos diferentes testes realizados pelas diferentes equipas nas quatro ilhas (valores de correlação intraclasse $-R$ ).

\begin{tabular}{lcccc} 
Variáveis & Faial & Pico & S. Miguel & Terceira \\
\hline Idade média (anos) & $8,23 \pm 1,37$ & $8,05 \pm 1,54$ & $8,06 \pm 1,42$ & $7,78 \pm 1,39$ \\
Amplitude etária & $6-12$ & $6-11$ & $6-10$ & $6-11$ \\
Equilíbrio retaguarda & 0,848 & 0,848 & 0,793 & 0,885 \\
Salto lateral & 0,857 & 0,867 & 0,932 & 0,889 \\
Transposição lateral & 0,878 & 0,889 & 0,870 & 0,776 \\
Salto monopedal & 0,887 & 0,898 & 0,981 & 0,921
\end{tabular}

Os resultados da avaliação da coordenação motora traduzem uma elevada fiabilidade. É mais do que evidente, dos resultados apresentados no quadro anterior, que estamos diante de informação de elevada qualidade, garantindo pois a análise subsequente dos dados e as ilações que deles venham a ser retiradas.

\section{RESULTADOS E DISCUSSÃO}

Os primeiros resultados referem-se às medidas descritivas básicas obtidas pelas meninas e pelos meninos em cada um dos testes da bateria KTK. Dada a dimensão amostral em cada intervalo etário, não ultrapassada por qualquer estudo realizado até ao momento em Portugal, os resultados encontrados podem servir de valores de referência, sobretudo para a população da Região Autónoma dos Açores, mas também, com as devidas ressalvas, para a população portuguesa em geral.

No Quadro 3 são apresentadas as medidas descritivas básicas dos resultados obtidos pelas meninas dos diferentes escalões etários nos testes da bateria KTK. 


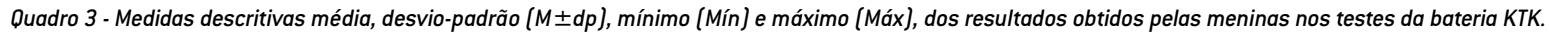

\begin{tabular}{|c|c|c|c|c|c|c|c|c|}
\hline \multirow{2}{*}{$\begin{array}{l}\text { Teste } \\
\text { Idade }\end{array}$} & \multicolumn{2}{|c|}{ ER } & \multicolumn{2}{|c|}{$\mathrm{TL}$} & \multicolumn{2}{|c|}{ SL } & \multicolumn{2}{|l|}{ SM } \\
\hline & $M \pm d p$ & Mín-Máx & $M \pm d p$ & Mín-Máx & $M \pm d p$ & Mín-Máx. & $M \pm d p$ & Mín-Máx \\
\hline 6 & $29,38 \pm 12,29$ & $1-65$ & $14,04 \pm 2,7$ & $8-22$ & $30,09 \pm 8,02$ & $8-60$ & $17,48 \pm 9,96$ & $0-51$ \\
\hline 7 & $32,69 \pm 13,28$ & $4-71$ & $14,95 \pm 3,08$ & $6-28$ & $35,33 \pm 10,98$ & $0-70$ & $23,44 \pm 11,69$ & $0-60$ \\
\hline 8 & $40,08 \pm 12,99$ & $4-71$ & $16,81 \pm 2,94$ & $7-25$ & $43,2 \pm 11,75$ & $3-77$ & $31,11 \pm 11,24$ & $0-59$ \\
\hline 9 & $42,31 \pm 12,97$ & $6-72$ & $18,05 \pm 3,22$ & $10-27$ & $48,74 \pm 11,59$ & $20-83$ & $35,41 \pm 13,75$ & 0.72 \\
\hline 10 & $44,13 \pm 13,68$ & $9-72$ & $18,79 \pm 3,22$ & $9-29$ & $51,7 \pm 12$ & $16-81$ & $40,83 \pm 14,33$ & $0-72$ \\
\hline
\end{tabular}

No Quadro 4 são apresentados os resultados, por idade, dos testes da bateria KTK, de meninas, obtidos em alguns estudos realizados em Portugal e no estrangeiro. Estes resultados vão servir-nos de referência para compararmos os valores médios obtidos pelas meninas da Região Autónoma dos Açores.
Devemos ressalvar, no entanto, que a amostra de qualquer dos estudos referidos nos quadros é inferior à amostra da presente investigação. A única excepção é o estudo de Kiphard e Schilling (5) que possui uma dimensão amostral bastante grande, mas, ainda assim, bastante inferior à da presente investigação.

Quadro 4 - Resultados ( $M \pm d p$ ), por idade, dos testes da bateria KTK de meninas obtidos em alguns estudos de referência realizados em Portugal e no estrangeiro.

\begin{tabular}{|c|c|c|c|c|c|}
\hline Idade & 6 & 7 & 8 & 9 & 10 \\
\hline \multicolumn{6}{|l|}{ Estudo } \\
\hline \multicolumn{6}{|l|}{ ER } \\
\hline Lopes (?] & & & $43,50 \pm 13,84$ & $41,07 \pm 14,50$ & \\
\hline Andrade (1) & & $45,68 \pm 11,02$ & $54,20 \pm 11,31$ & $58,50 \pm 9,20$ & \\
\hline Kiphard e Schilling (5) & $37,20 \pm 11,00$ & $42,70 \pm 13,10$ & $48,20 \pm 13,90$ & $52,10 \pm 13,20$ & $58,50 \pm 20,10$ \\
\hline Gomes (2) & & & $44,00 \pm 9,90$ & $47,10 \pm 9,90$ & $47,30 \pm 12,00$ \\
\hline \multicolumn{6}{|l|}{$\overline{S M}$} \\
\hline Lopes (?) & & & $35,12 \pm 12,74$ & $36,27 \pm 13,26$ & \\
\hline Andrade (1] & & $35,92 \pm 13,98$ & $46,82 \pm 11,31$ & $53,43 \pm 10,30$ & \\
\hline Kiphard e Schilling (5) & $34,40 \pm 10,40$ & $37,30 \pm 11,80$ & $42,60 \pm 13,10$ & $54,80 \pm 14,30$ & $63,10 \pm 10,10$ \\
\hline Gomes (2) & & & $34,50 \pm 10,30$ & $41,10 \pm 13,10$ & $39,80 \pm 12,80$ \\
\hline \multicolumn{6}{|l|}{$\overline{S L}$} \\
\hline Lopes (?] & & & $51,54 \pm 12,96$ & $57,00 \pm 12,26$ & \\
\hline Andrade (1) & & $37,71 \pm 10,79$ & $48,34 \pm 9,40$ & $55,02 \pm 10,00$ & \\
\hline Kiphard e Schilling (5) & $37,30 \pm 9,20$ & $43,20 \pm 10,20$ & $48,00 \pm 11,70$ & $60,80 \pm 19,50$ & $67,20 \pm 10,70$ \\
\hline Gomes (2) & & & $47,30 \pm 9,80$ & $54,00 \pm 11,80$ & $55,50 \pm 12,50$ \\
\hline \multicolumn{6}{|l|}{$\mathrm{TL}$} \\
\hline Lopes (?] & & & $16,73 \pm 3,35$ & $19,73 \pm 4,27$ & \\
\hline Andrade (1) & & $17,44 \pm 3,28$ & $19,57 \pm 3,12$ & $21,97 \pm 3,82$ & \\
\hline Kiphard e Schilling (5) & $32,60 \pm 4,30$ & $36,20 \pm 5,90$ & $38,80 \pm 5,80$ & $44,30 \pm 6,20$ & $47,90 \pm 5,90$ \\
\hline Gomes [2] & & & $16,30 \pm 2,70$ & $17,40 \pm 3,06$ & $18,00 \pm 2,80$ \\
\hline
\end{tabular}


Os resultados obtidos pelas meninas merecem os seguintes comentários:

- No teste de equilíbrio em marcha à retaguarda (ER) os resultados das crianças da Região Autónoma dos Açores são, em todas as idades, mais baixos do que os estudos de referência. Os valores dos desvios-padrão são de magnitude reduzida, o que indica alguma concentração dos resultados individuais em redor do valor central (média), não havendo, portanto, grande variabilidade inter-individual, com a excepção do grupo etário de 6 anos de idade, onde atinge quase metade do valor da média.

- No teste de transposição lateral (TL) a situação é semelhante, sendo o valor médio das crianças da Região Autónoma dos Açores, em todos os intervalos etários, menos de metade dos valores médios das crianças do estudo de Kiphard e Schilling (5). - No teste de saltos laterais (SL) os valores das crianças da Região Autónoma dos Açores são também mais baixos do que os valores encontrados nos outros estudos em todos os escalões etários.

- No teste de saltos monopedais (SM) o quadro de resultados é idêntico aos anteriores. Verifica-se a existência, em todos os intervalos etários, de crianças com valores de prova igual a zero, sendo os valores máximos bastante elevados, o que, conjuntamente com os valores verificados nos desvios-padrão, nos leva a referir a existência de variabilidade interindividual elevada.

No Quadro 5 são apresentadas as medidas descritivas básicas dos resultados obtidos pelos meninos dos diferentes escalões etários nos testes da bateria KTK.

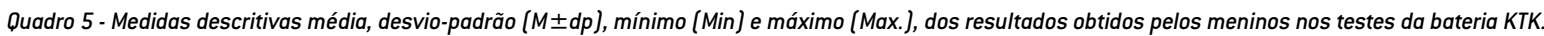

\begin{tabular}{|c|c|c|c|c|c|c|c|c|}
\hline \multirow{2}{*}{$\begin{array}{l}\text { Teste } \\
\text { Idade }\end{array}$} & \multicolumn{2}{|c|}{ ER } & \multicolumn{2}{|c|}{ TL } & \multicolumn{2}{|c|}{ SL } & \multicolumn{2}{|c|}{ SM } \\
\hline & $M \pm d p$ & Mín-Máx & $M \pm d p$ & Mín-Máx & $M \pm d p$ & Mín-Máx. & $M \pm d p$ & Mín-Máx \\
\hline 6 & $29,21 \pm 11,94$ & $0-61$ & $14,86 \pm 2,84$ & $8-24$ & $30,93 \pm 8,67$ & $11-60$ & $17,96 \pm 10,96$ & $0-53$ \\
\hline 7 & $35,4 \pm 13,03$ & $3-72$ & $15,83 \pm 3,17$ & $6-26$ & $35,44 \pm 9,44$ & $9-75$ & $26,69 \pm 12,78$ & $0-65$ \\
\hline 8 & $40,64 \pm 13,47$ & $4-72$ & $17,55 \pm 3,36$ & $5-28$ & $41,7 \pm 11,93$ & $4-74$ & $34,2 \pm 14,28$ & $0-68$ \\
\hline 9 & $45,13 \pm 13,36$ & $5-72$ & $19,07 \pm 3,58$ & $8-31$ & $46,98 \pm 12,37$ & $9-83$ & $41,09 \pm 13,77$ & 0.72 \\
\hline 10 & $49,64 \pm 13,5$ & $10-72$ & $20,64 \pm 3,48$ & $12-30$ & $51,33 \pm 12,59$ & $18-88$ & $46,92 \pm 15,18$ & $0-72$ \\
\hline
\end{tabular}

No Quadro 6 são apresentados os resultados, por idade, dos testes da bateria KTK, de meninos, obtidos em alguns estudos realizados em Portugal e no estrangeiro. Tal como nas meninas, estes dados ser- vem-nos de referência para compararmos os valores médios obtidos pelos meninos da Região Autónoma dos Açores. 
Quadro 6 - Resultados ( $M \pm d p$ ), por idade, dos testes da bateria KTK de meninos obtidos em alguns estudos de referência realizados em Portugal e no estrangeiro.

\begin{tabular}{|c|c|c|c|c|c|}
\hline Idade & 6 & 7 & 8 & 9 & 10 \\
\hline \multicolumn{6}{|l|}{ Estudo } \\
\hline \multicolumn{6}{|l|}{ ER } \\
\hline Lopes (?] & & & $46,00 \pm 12,17$ & $51,64 \pm 14,65$ & \\
\hline Andrade (1) & & $47,81 \pm 9,59$ & $48,68 \pm 12,77$ & $54,26 \pm 9,90$ & \\
\hline Kiphard e Schilling (5) & $31,30 \pm 15,00$ & $39,90 \pm 13,90$ & $46,90 \pm 13,60$ & $51,10 \pm 15,30$ & $54,30 \pm 13,00$ \\
\hline Gomes (2) & & & $45,10 \pm 12,00$ & $50,01 \pm 9,70$ & $51,20 \pm 12,60$ \\
\hline \multicolumn{6}{|l|}{$\overline{S M}$} \\
\hline Lopes (?) & & & $39,70 \pm 11,46$ & $46,56 \pm 11,90$ & \\
\hline Andrade [1] & & $38,73 \pm 12,21$ & $49,42 \pm 11,54$ & $56,80 \pm 10,56$ & \\
\hline Kiphard e Schilling (5) & $23,80 \pm 14,50$ & $34,40 \pm 13,60$ & $45,20 \pm 14,20$ & $52,60 \pm 15,20$ & $58,70 \pm 14,0$ \\
\hline Gomes (2) & & & $37,90 \pm 13,10$ & $47,00 \pm 10,20$ & $48,90 \pm 12,60$ \\
\hline \multicolumn{6}{|l|}{$\overline{S L}$} \\
\hline Lopes (?) & & & $45,09 \pm 10,39$ & $52,44 \pm 10,75$ & \\
\hline Andrade (1) & & $40,52 \pm 8,83$ & $44,52 \pm 7,93$ & $49,49 \pm 12,36$ & \\
\hline Kiphard e Schilling (5) & $31,30 \pm 12,70$ & $36,50 \pm 10,00$ & $45,80 \pm 11,70$ & $50,40 \pm 13,20$ & $59,40 \pm 11,60$ \\
\hline Gomes [2] & & & $48,30 \pm 12,50$ & $52,50 \pm 10,30$ & $54,50 \pm 11,00$ \\
\hline \multicolumn{6}{|l|}{$\overline{\mathrm{TL}}$} \\
\hline Lopes (?) & & & $18,57 \pm 3,92$ & $20,68 \pm 3,53$ & \\
\hline Andrade (1) & & $18,73 \pm 2,64$ & $20,24 \pm 2,90$ & $22,02 \pm 3,06$ & \\
\hline Kiphard e Schilling (5) & $31,90 \pm 8,20$ & $35,50 \pm 6,60$ & $40,60 \pm 6,20$ & $43,00 \pm 7,70$ & $46,40 \pm 6,00$ \\
\hline Gomes [2] & & & $17,80 \pm 2,70$ & $19,30 \pm 3,60$ & $20,10 \pm 3,50$ \\
\hline
\end{tabular}

Os resultados obtidos pelos meninos merecem os seguintes comentários:

- No teste de equilíbrio em marcha à retaguarda (ER) os resultados das crianças da Região Autónoma dos Açores são inferiores aos resultados referidos nos outros estudos. No intervalo etário de 6 anos de idade existem crianças que obtêm uma pontuação igual a zero. Nos outros intervalos etários existem crianças que obtêm a pontuação máxima (72 pontos).

- No teste de transposição lateral (TL) a situação é semelhante, sendo o valor médio das crianças da Região Autónoma dos Açores, em todos os intervalos etários, tal como nas meninas, menos de metade dos valores médios das crianças do estudo de Kiphard e Schilling (5).

- No teste de saltos laterais os valores médios das crianças da Região Autónoma dos Açores são, mais uma vez, inferiores aos obtidos nos outros estudos.
- No teste de saltos monopedais (SM) o quadro de resultados é idêntico aos anteriores. Verifica-se, tal como nas meninas, a existência, em todos os intervalos etários, de crianças com valores de prova igual a zero, sendo os valores máximos bastante elevados. O que, tendo também em consideração os valores relativamente elevados nos desvios-padrão, nos leva a referir a existência de uma elevada variabilidade inter-individual.

\section{Comparação entre sexos em função da idade}

A Figura 1 refere-se ao comportamento dos valores médios dos testes da bateria KTK em função da idade e do sexo. O Quadro 7 apresenta os resultados da ANOVA de dois factores (sexo * idade) às diferenças entre os sexos e os escalões etários em cada teste do KTK. 

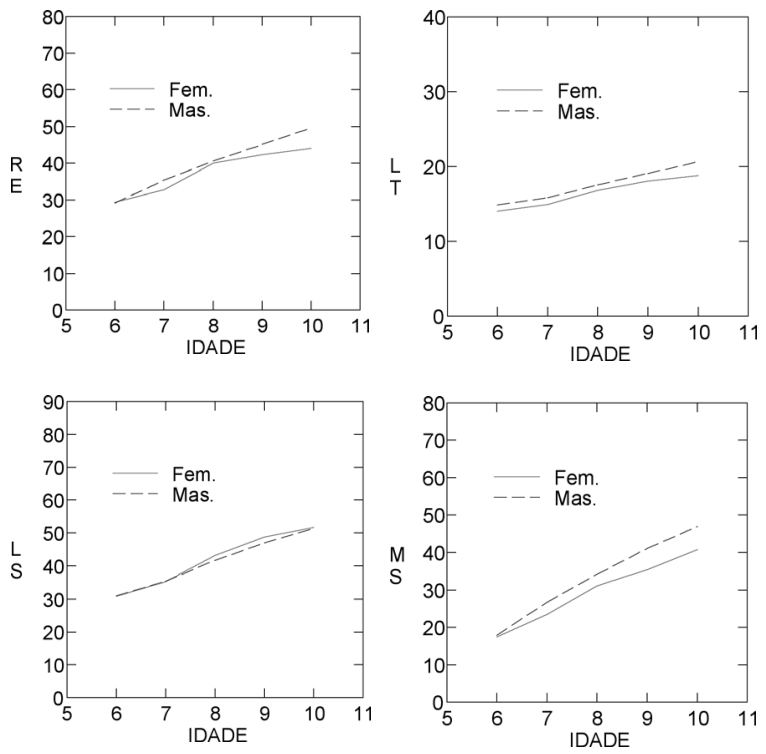

Figura 1 - Comportamento dos valores médios dos testes da bateria KTK em função da idade e do sexo.

Quadro 7 - Resultados da ANOVA de dois factores [sexo * idade) às diferenças entre os sexos e escalões etários em cada teste do KTK.

\begin{tabular}{|c|c|c|c|}
\hline Teste & Factor & $F(g \mid)$ & $\mathrm{p}$ \\
\hline \multicolumn{4}{|l|}{ ER } \\
\hline & Sexo & $26,765(1,3731)$ & $<0,001$ \\
\hline & Idade & $185,912(4,3731)$ & $<0,001$ \\
\hline & Sexo $*$ Idade & $4,280[4,3731]$ & 0,002 \\
\hline \multicolumn{4}{|l|}{$\overline{\mathrm{TI}}$} \\
\hline & Sexo & $97,397(1,3727)$ & $<0,001$ \\
\hline & Idade & $292,875(4,3727)$ & $<0,001$ \\
\hline & Sexo $*$ Idade & $2,885(4,3727)$ & 0,021 \\
\hline \multicolumn{4}{|l|}{$\mathrm{SI}$} \\
\hline & Sexo & $3,464(1,3727)$ & ns \\
\hline & Idade & $370,646(4,3727)$ & $<0,001$ \\
\hline & Sexo $*$ Idade & $1,252(4,3727)$ & ns \\
\hline \multicolumn{4}{|l|}{$\overline{S M}$} \\
\hline & Sexo & $72,616(1,3732)$ & $<0,001$ \\
\hline & Idade & $393,414(4,3732)$ & $<0,001$ \\
\hline & Sexo $*$ Idade & $4,751(4,3732)$ & 0,001 \\
\hline
\end{tabular}

Com a excepção do teste saltos laterais (SL) os meninos apresentam valores médios superiores aos das meninas em todos os intervalos etários. Estas diferenças são estatisticamente significativas conforme se pode ver nos resultados da ANOVA (Quadro 7). No teste SL não se verificam diferenças estatisticamente significativas. Apenas aos 8 e aos 9 anos de idade os meninos apresentam valores médios superiores. Nos outros intervalos etários os resultados dos dois sexos são semelhantes. Esta diferença no nível de desenvolvimento da coordenação motora entre os meninos e as meninas pode ficar a dever-se a diferentes oportunidades de prática de actividade físico-motora, sobretudo no meio familiar. É conhecido que no meio familiar, sobretudo onde o papel dos sexos está bem vincado e arreigado a tradições, as oportunidades e o tipo de brincadeiras permitidas às crianças dos dois sexos são distintas (sobre este assunto ver 8). Estes resultados são semelhantes aos encontrados por Willimczik (14) que verificou, num estudo longitudinal realizado com crianças seguidas dos 6 aos 10 anos de idade, que, embora as meninas aos seis anos de idade apresentassem melhor níveis de coordenação do que os meninos, a partir do 8 anos de idade os meninos mostravam resultados superiores às meninas. Em contradição com estes resultados estão os resultados encontrados por Kiphard e Schilling (5), que verificaram que as meninas tinham prestações superiores aos meninos em todos os níveis etários entre os 6 e os 10 anos de idade.

É notório o incremento dos valores médios ao longo da idade em ambos os sexos, em todos os testes da bateria KTK. Este incremento é estatisticamente significativo em todos os testes (Quadro 7). A magnitude do incremento é superior nos rapazes relativamente às raparigas em todos os testes à excepção do teste SL, em que não se verificou interacção significativa entre os factores sexo e idade (Quadro 7). Estes resultados são semelhantes aos encontrados por Kiphard e Schilling (5) no que diz respeito ao crescimento dos valores médios ao longo da idade, já que não ocorre um crescimento paralelo da prestação dos dois sexos, tal como Kiphard e Schilling (5) verificaram. 


\section{Quociente motor}

No Quadro 8 são apresentadas as medidas descritivas básicas do QM, nas diferentes idades, das meninas e dos meninos.

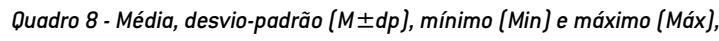
do $Q M$ nas diferentes idades em ambos os sexos.

\begin{tabular}{lcccc} 
& \multicolumn{2}{c}{ Meninas } & \multicolumn{2}{c}{ Meninos } \\
Idade & $M \pm d p$ & Mín-Máx & M dp & Mín-Máx \\
\hline 6 & $73,86 \pm 10,82$ & $49-111$ & $82,42 \pm 10,42$ & $55-116$ \\
7 & $72,9 \pm 12,57$ & $40-108$ & $80,21 \pm 11,8$ & $45-113$ \\
8 & $75,42 \pm 11,29$ & $40-106$ & $76,59 \pm 12,71$ & $40-108$ \\
9 & $68,44 \pm 12,38$ & $40-105$ & $76,99 \pm 11,76$ & $40-113$ \\
10 & $65,88 \pm 12,23$ & $40-97$ & $75,26 \pm 12,98$ & $40-109$
\end{tabular}

Os valores das médias são baixos, tanto nos meninos como nas meninas. Quer nas meninas, quer nos meninos, verifica-se um decréscimo significativo do $\mathrm{QM}$ ao longo da idade $[\mathrm{F}(4,3732)=40,62, \mathrm{P}<0,001]$. As meninas apresentam valores médios significativamente inferiores aos meninos $[\mathrm{F}(1,3732)=299,80$, $\mathrm{P}<0,001]$. Nos meninos verifica-se que, em todas as idades, os valores médios do QM se situam no intervalo de insuficiência coordenativa. Nas meninas, aos 6, 7 e 8 anos de idade, os valores médios situam-se no intervalo de insuficiência coordenativa, aos 9 e aos 10 anos de idade os valores médios situam-se no intervalo de perturbações de coordenação.

No Quadro 9 e no Quadro 10 são apresentadas as distribuições das categorias de nível de desenvolvimento coordenativo por idades, nas meninas e nos meninos, e por ilhas.

Quadro 9 - Distribuição das categorias de nível de desenvolvimento coordenativo por estratos etários nas meninas e nos meninos.

\begin{tabular}{|c|c|c|c|c|c|c|c|}
\hline & & & & Níveis d & volvime & rdenat & \\
\hline & Idade & & 1 & 2 & 3 & 4 & 5 \\
\hline Meninas & 6 & Número & 106 & 125 & 38 & & \\
\hline & & $\%$ & $39,4 \%$ & $46,5 \%$ & $14,1 \%$ & & \\
\hline & 7 & Número & 190 & 175 & 66 & & \\
\hline & & $\%$ & $44,1 \%$ & $40,6 \%$ & $15,3 \%$ & & \\
\hline & 8 & Número & 143 & 195 & 90 & & \\
\hline & & $\%$ & $33,4 \%$ & $45,6 \%$ & $21,0 \%$ & & \\
\hline & 9 & Número & 260 & 161 & 39 & & \\
\hline & & $\%$ & $56,5 \%$ & $35,0 \%$ & $8,5 \%$ & & \\
\hline & 10 & Número & 148 & 88 & 5 & & \\
\hline & & $\%$ & $61,4 \%$ & $36,5 \%$ & $2,1 \%$ & & \\
\hline & Total & Número & 847 & 744 & 238 & 0 & 0 \\
\hline & & $\%$ & $46,3 \%$ & $40,7 \%$ & $13,0 \%$ & $0 \%$ & $0 \%$ \\
\hline Meninos & 6 & Número & 35 & 146 & 104 & 1 & \\
\hline & & $\%$ & $12,2 \%$ & $51,0 \%$ & $36,4 \%$ & $0,3 \%$ & \\
\hline & $?$ & Número & 83 & 228 & 142 & & \\
\hline & & $\%$ & $18,3 \%$ & $50,3 \%$ & $31,3 \%$ & & \\
\hline & 8 & Número & 136 & 203 & 125 & & \\
\hline & & $\%$ & $29,3 \%$ & $43,8 \%$ & $26,9 \%$ & & \\
\hline & 9 & Número & 131 & 185 & 113 & & \\
\hline & & $\%$ & $30,5 \%$ & $43,1 \%$ & $26,3 \%$ & & \\
\hline & 10 & Número & 90 & 129 & 62 & & \\
\hline & & $\%$ & $32,0 \%$ & $45,9 \%$ & $22,1 \%$ & & \\
\hline & Total & Número & 475 & 891 & 546 & 1 & 0 \\
\hline & & $\%$ & $24,8 \%$ & $46,6 \%$ & $28,5 \%$ & $0,1 \%$ & $0 \%$ \\
\hline
\end{tabular}


Os valores apresentados nos quadros anteriores são portadores de alguma inquietação, não devendo deixar ninguém indiferente perante uma situação tão negativa dos níveis de desenvolvimento coordenativo das crianças da Região Autónoma dos Açores.

Verifica-se que a generalidade das crianças de ambos os sexos nos diferentes intervalos etários se situam no intervalo de insuficiência coordenativa e de perturbações de coordenação. Uma percentagem muito reduzida de crianças situa-se no intervalo de coordenação normal, atingindo um valor máximo de 36,4\% aos 6 anos nos meninos. Nenhuma menina se situa no intervalo de coordenação boa ou muito boa.
Apenas um menino se situa no intervalo de coordenação boa; nenhum se situa no intervalo de coordenação muito boa.

A ilha das Flores é aquela que apresenta uma maior percentagem de crianças no nível de perturbações de coordenação (50\%), seguida da ilha de Santa Maria (47,5\%). Considerando conjuntamente os níveis de perturbações de coordenação e de insuficiência coordenativa, verifica-se que, em todas as ilhas, mais de metade das crianças se situam num ou noutro nível. De facto, em nenhuma ilha o valor percentual do intervalo de coordenação normal atinge os $50 \%$. O valor mais elevado é de $46,4 \%$ na ilha do Pico.

Quadro 10 - Distribuição das categorias de nível de desenvolvimento coordenativo por ilhas.

\begin{tabular}{|c|c|c|c|c|c|c|c|c|}
\hline & & & Níveis & volvimen & denativo & & & \\
\hline & & & 1 & 2 & 3 & 4 & 5 & Total \\
\hline ILHA & Faial & Número & 46 & 63 & 23 & & & 132 \\
\hline & & $\%$ & $34,8 \%$ & $47,7 \%$ & $17,4 \%$ & & & $100,0 \%$ \\
\hline & Flores & Número & 31 & 18 & 13 & & & 62 \\
\hline & & $\%$ & $50,0 \%$ & $29,0 \%$ & $21,0 \%$ & & & $100,0 \%$ \\
\hline & Graciosa & Número & 12 & 25 & 24 & & & 61 \\
\hline & & $\%$ & $19,7 \%$ & $41,0 \%$ & $39,3 \%$ & & & $100,0 \%$ \\
\hline & Pico & Número & 26 & 63 & 77 & & & 166 \\
\hline & & $\%$ & $15,7 \%$ & $38,0 \%$ & $46,4 \%$ & & & $100,0 \%$ \\
\hline & Santa Maria & Número & 56 & 45 & 17 & & & 118 \\
\hline & & $\%$ & $47,5 \%$ & $38,1 \%$ & $14,4 \%$ & & & $100,0 \%$ \\
\hline & São Jorge & Número & 54 & 65 & 32 & & & 151 \\
\hline & & $\%$ & $35,8 \%$ & $43,0 \%$ & $21,2 \%$ & & & $100,0 \%$ \\
\hline & São Miguel & Número & 866 & 1050 & 426 & 1 & & 2343 \\
\hline & & $\%$ & $37,0 \%$ & $44,8 \%$ & $18,2 \%$ & $0,0 \%$ & & $100,0 \%$ \\
\hline & Terceira & Número & 231 & 306 & 172 & & & 709 \\
\hline & & $\%$ & $32,6 \%$ & $43,2 \%$ & $24,3 \%$ & & & $100,0 \%$ \\
\hline Total & & Número & 1322 & 1635 & 784 & 1 & & 3742 \\
\hline & & $\%$ & $35,3 \%$ & $43,7 \%$ & $21,0 \%$ & $0,0 \%$ & $0 \%$ & $100,0 \%$ \\
\hline
\end{tabular}

Os resultados verificados levantam a questão da adequabilidade das tabelas normativas que levam ao cálculo do QM. De facto, numa amostra tão vasta como a do presente estudo, e representativa da população escolar dos Açores, parece-nos pouco provável não haver crianças com níveis de coordenação bons e mesmo muito bons. Os resultados obtidos por
Mjaavatn et al. (10) parecem dar alguma consistência a esta hipótese. Os autores verificaram que nenhuma das crianças da amostra (quatro escolas básicas da Noruega) se situava nos níveis de coordenação bom e muito bom. Pode também colocar-se a questão da validade transcultural dos valores normativos e dos valores de corte utilizados para a classifi- 
cação das crianças. A bateria de testes KTK e os estudos que levaram ao desenvolvimento das tabelas normativas foram realizados na Alemanha, com crianças alemãs. É, portanto, provável haver algum desajustamento dos valores de corte relativamente à população açoreana.

\section{Análise aos perfis multivariados da coordenação motora}

Se considerarmos, não a análise de cada teste individualmente, nem o QM resultante, mas pensarmos que os valores que cada criança obteve nos 4 testes de coordenação definem um perfil multidimensional da configuração da sua coordenação motora, seria lícito colocar a seguinte pergunta:

- Será que o perfil configuracional da coordenação motora das crianças corresponde aquele que é esperado para a sua idade?

É evidente que no domínio lato do desenvolvimento motor é de esperar alguma heterogeneidade interindividual. Mas também é correcto pensar, de acordo com as posições normativistas, que, no seio de cada idade, as crianças evidenciam um perfil de coordenação que as torna homogénas, i.e., em cada idade haverá um tipo de coordenação motora esperada. Se, porventura, os valores dos perfis das crianças na sua coordenação forem diferentes daquele que é esperado, então estamos diante de um de dois problemas:

(1) se os perfis corresponderem aos de crianças mais velhas, então teremos que redimensionar aspectos da estrutura didáctico-metodológica das aulas face ao valor mais elevado da prontidão coordenativa das crianças; (2) o contrário é que se afigura mais problemático, se constatarmos que os perfis das crianças correspondem aos perfis esperados de crianças de idades mais baixas. Estamos aqui num domínio delicado, e que coloca o problema de insuficiência de prontidão coordenativa. Esta matéria exige pois um cuidado acrescido na própria elaboração dos planos das aulas para respeitar, em cada idade, a enorme variedade dos níveis de prontidão coordenativa das crianças.

Os valores desta matriz de reclassificação estão no Quadro 11 e no Quadro 12, respectivamente, para as meninas e para os meninos.
Quadro 11 - Tabela de reclassificação das meninas (frequências relativas) com base nos resultados significativos da função discriminante (FD) encontrada. A negrito encontram-se os números respeitantes às meninas correctamente reclassificadas nas suas idades (i.e. com um perfil de coordenação esperado para a sua idade).

Idades Grupos naturais de idade [previsão com base nos resultados da FD]

\begin{tabular}{l|llllll}
\hline \multicolumn{1}{c}{} & 6 & $?$ & 8 & 9 & 10 & Total \\
\cline { 2 - 7 } 6 & $\mathbf{6 3 , 1}$ & 24,6 & 8,6 & 1,9 & 1,9 & 100 \\
7 & 38,7 & $\mathbf{2 8 , 3}$ & 14,6 & 10,4 & 7,9 & 100 \\
8 & 12,1 & 20,3 & $\mathbf{2 6 , 6}$ & 20,3 & 20,6 & 100 \\
9 & 8,5 & 11,5 & 18,7 & $\mathbf{2 3 , 9}$ & 37,4 & 100 \\
10 & 6,7 & 7,5 & 13,4 & 18,4 & $\mathbf{5 4 , 0}$ & 100
\end{tabular}

Se atentarmos na percentagem de reclassificação das meninas nos seus grupos naturais, i.e., a sua idade cronológica, com base nos seus perfis multidimensionais de aptidão física verificamos que:

- Aos 6 anos a percentagem é de $63,1 \%$ bem classificados. A partir desta idade, a percentagem é baixíssima: $28,3 \%$ aos 7 anos, $26,6 \%$ aos 8 anos, $23,9 \%$ aos 9 anos e $54 \%$ aos 10 anos. Trata-se, sem qualquer sombra de dúvida, de valores muito baixos. - É claro que há meninas que na sua idade revelam um perfil de coordenação motora que caracteriza as suas colegas de idades mais avançadas, como é, por exemplo, o caso dos 6 anos, em que há 66 meninas com perfis de coordenação motora de meninas de 7 anos e assim por diante. Do mesmo modo perceberemos esta coordenação avançada nas meninas de 7 , 8 e 9 anos de idade.

- Contudo, aquilo que de certo modo evidencia algo de preocupante, é verificar a presença de crianças cujo perfil corresponde ao das suas colegas de idade mais baixa. Por exemplo, aos 7 anos há 167 meninas que possuem um perfil de aptidão de colegas de 6 anos de idade! $\mathrm{E}$ a leitura estende-se às de 8 e 9 anos de idade. Aos 10 anos de idade, há mais meninas com perfis de colegas de idade mais baixa, do que aquelas que seriam esperadas para a sua idade. Ora este é, sem dúvida, um problema sério que necessita uma análise cuidada dos perfis de coordenação motora, e, concretamente, da prontidão motora das crianças tendo em conta que se espera que as aulas de Educação Física produzam algum efeito. 
Quadro 12 - Tabela de reclassificação dos meninos (frequências relativas) com base nos resultados significativos da função discriminante (FD) encontrada. A negrito encontram-se os números respeitantes aos meninos correctamente reclassificados nas suas idades (i.e. com um perfil de coordenação esperado para a sua idade].

Idades Grupos naturais de idade (previsão com base nos resultados da FD)

\begin{tabular}{l|llllll}
\hline \multicolumn{1}{c}{} & 6 & 7 & 8 & 9 & 10 & Total \\
\cline { 2 - 7 } 6 & $\mathbf{6 7 , 0}$ & 19,6 & 9,5 & 2,8 & 1,1 & 100 \\
7 & 34,7 & $\mathbf{3 0 , 1}$ & 18,6 & 11,9 & 4,6 & 100 \\
8 & 17,9 & 17,1 & $\mathbf{2 3 , 5}$ & 20,7 & 20,7 & 100 \\
9 & 7,2 & 13,8 & 16,1 & $\mathbf{2 1 , 0}$ & 41,8 & 100 \\
10 & 6,4 & 5,3 & 10,0 & 14,2 & $\mathbf{6 4 , 1}$ & 100
\end{tabular}

Tal como anteriormente, este quadro de resultados necessita uma leitura muito atenta por parte de professores de Educação Física e professores(as) envolvidos(as) nas aulas curriculares destas crianças no que à leccionação da Educação Física diz respeito. Tal como anteriormente, a interpretação dos números conduz a um cuidado substancial na determinação inicial dos níveis de prontidão motora das crianças, por forma a que o planeamento das aulas, concretamente a sua estrutura didáctico-metodológica, contemple, em cada ano de escolaridade, a forte heterogeneidade patente dos níveis de desenvolvimento motor das crianças.

- Aos 6 anos a percentagem de meninos é de $67 \%$ (191 meninos em 285) bem classificados. A partir desta idade, a percentagem é baixíssima: 29,2\% (132 meninos) aos 7 anos, 24\% (111 meninos) aos 8 anos, $21 \%$ (90 meninos) aos 9 anos e 65,8\% (185 meninos) aos 10 anos. Trata-se, sem dúvida, de valores muito baixos de reclassificação dos meninos nos seus grupos originais de idade. O que é mais saliente é, sem dúvida, a sua "má reclassificação".

-É claro que há meninos que na sua idade revelam um perfil de coordenação motora que caracteriza os seus colegas de idades mais avançadas, como são, por exemplo, os casos dos 6 aos 9 anos em que há números "substanciais" de crianças cujos perfis de coordenação motora as colocam noutras idades. - Contudo, aquilo que nos preocupa é verificar a presença de crianças cujo perfil corresponde ao dos seus colegas de idade mais baixa. Por exemplo, aos 7 anos há 158 meninos que possuem um perfil de aptidão de colegas de 6 anos de idade! E a leitura estende-se às de 8 e 9 anos de idade. Aos 9 anos há cerca de 154 meninos (36\%) cujos perfis multidimensionais de aptidão os colocam em idades mais baixas! Aos 10 anos de idade, há mais meninos com perfis de colegas de idade mais baixa, do que aqueles que seriam esperados para a sua idade.

Os resultados desta análise, embora não confirmem plenamente os resultados obtidos no QM, também não os infirmam.

Sendo estes resultados tão negativos, deveriam as autoridades educativas da Região Autónoma dos Açores tomar medidas sérias para a remediação desta "epidemia" que é o fraco nível de desenvolvimento coordenativo das crianças do 1CEB.

\section{CONCLUSÕES}

Em todas as provas de coordenação, e ao longo da idade, verifica-se um incremento significativo dos valores médios.

Os valores médios do desempenho nas quatro provas do KTK das crianças açoreanas são inferiores aos obtidos noutros estudos realizados, quer em Portugal, quer no estrangeiro.

A generalidade das crianças são identificadas como possuindo níveis de desenvolvimento coordenativo muito baixos. Nas meninas verifica-se que $46,3 \%$ são classificadas como possuindo perturbações de coordenação e $40,7 \%$ como possuindo insuficiência coordenativa. Nos meninos constata-se que $24,8 \%$ são classificadas como possuindo perturbações de coordenação e $46,6 \%$ como possuindo insuficiência coordenativa.

Verifica-se uma tendência generalizada para as meninas de uma dada idade mostrarem perfis de coordenação motora inferiores aqueles que são esperados para a sua idade. Tal circunstância revela uma forte insuficiência em aspectos do desenvolvimento coordenativo nas diferentes idades.

*Financiado pela Direcção Regional de Educação Física e Desporto e pela Direç̧ão Regional da Ciência e Tecnologia da Região Autónoma dos Açores. 


\section{CORRESPONDÊNCIA}

Vítor Pires Lopes

Escola Superior de Educação de Bragança

Campus de Santa Apolónia

Apartado 1101

5300-850 Bragança

vplopes@ipb.pt

\section{BIBLIOGRAFIA}

1 Andrade, M. J. L. A. (1996). Coordenação motora. Estudo em crianças do ensino básico na Região Autónoma da Madeira. Dissertação de mestrado. Porto: FCDEF-UP (não publicada).

2 Gomes, M. P. B. B. (1996). Coordenação, aptidão física e variáveis do envolvimento. Estudo em crianças do $1^{\circ}$ ciclo de ensino de duas freguesias do concelho de Matosinhos. Tese de doutoramento. Porto: FCDEF-UP (não publicada).

3 Kiphard, E. J. (1976). Insuficiencias de movimiento y de coordinación en la edad de la escuela primaria. Buenos Aires: Editorial Kapelusz.

4 Kiphard, E. J.; Schilling, F. (1970) Der hamm-marburgerkoordinationstest für kinder (HMKTK). Monatszeitsschrift für Kinderheit Kunde 118 (6): 473-479.

5 Kiphard, E. J; Schilling, F. (1974). Körperkoordinationstest für kinder, KTK. Weinheim: Beltz.

6 Leurs, S.; Dordel, S.; Lawewnz, W.; Schichendantz, S.; Stiker, E.; Bjarnason-Wehrens, B. (2001). The importance of a psychomotor training for children with congenital heart disease (CHD) in the age of 7-14 years. In: J. Mester, G. King, H. Struder, E. Tsolakidis, A. Osterburg (eds.) Book of Abstracts of 6th Annual Congress of the European College of Sport Science. Cologne: Sport und Buch Strauß, 631.

7 Lopes, V. P. (1997). Análise dos efeitos de dois programas distintos de educação física na expressão da aptidão física, coordenação e habilidades motoras em crianças do ensino primário. Tese de Doutoramento. Porto: FCDEF-UP (não publicada).

8 Lopes, V. P. (1998). Indicadores Bioculturais e Somáticos do Rendimento Motor em Crianças de 5/6 anos de Idade. Série estudos, n. ${ }^{\circ}$ 33. Bragança: Instituto Politécnico de Bragança.

9 Lopes, V. P.; Maia, J. A. R. (1997). Efeitos do ensino no desenvolvimento da capacidade de coordenação corporal em crianças de 8 anos de idade. Revista Paulista de Educação Física. 11(1): 40-48.

10 Mjaavatn, P. E.; Gundersen, K. A.; Segberg, V.; Bjorkenlund, L. A. (2003). Physical activity and healthrelated variables in 6-9 year-old Norwegian children. Medicine and Science in Sport and Exercise. 35(5): S63.

11 Martinek, T. J.; Zaichkowsky, L. D.; Cheffers, J. T. F. (1977). Decision-making in elementary age children: effects on motor skills and self-concept. Res. Q. Exerc. Sport. 48 (2):349-356.

12 Meinel, K.; Schnabel, G. (1984). Motricidade I. Teoria da motricidade esportiva sob o aspecto pedagógico. Rio de Janeiro: Ao Livro Técnico.

13 Mota, J. A. P. S. (1991). Contributo para o desenvolvimento de programas de aulas suplementares de educação física. Estudo experimental em crianças com insuficiências de rendimento motor. Tese de doutoramento. Porto: FCDEFUP (não publicada).

14 Willimczik, K. (1980). Development of motor control capability (body coordination) of 6-to 10-year-old children: Results of a Longitudinal Study. In M. Ostyn; G. Beunen; J. Simons (eds.). Kinanthropometry II. Baltimore: University Park Press.

15 Zaichkowsky, L. D.; Zaichkowsky, L. B.; Martinek, T. J. (1978). Physical Activity, motor development age and sex differences. In: Landry, F; Orban, W. D. R. (eds) Motor learning, sport psychology, pedagogy and didactics of physical activity. Miami: Symposia Specialists 